Original Article

\title{
Prospective Study of Sarpogrelate Hydrochloride on Patients with Arteriosclerosis Obliterans
}

\author{
Shiyan Ren, MD, PhD, ${ }^{1}$ Songyi Qian, MD,${ }^{1}$ Wei Wang, MSc, ${ }^{2}$ Fei Wang, MD,${ }^{1}$ \\ Jiagtao Liu, MD, ${ }^{1}$ and Peng Liu, MD, $\mathrm{PhD}^{1}$
}

\begin{abstract}
Objective: To evaluate the effect of sarpogrelate for patients with atherosclerotic obliterans (ASO).

Patients and Methods: Patients with ASO were randomly divided into sarpogrelate group $(n=92)$ and control group $(n=84)$. The patients in sarpogrelate group received sarpogrelate (100 $\mathrm{mg}$, tid), whereas in control group aspirin $(100 \mathrm{mg}$, qd) was administered orally. The patients were followed up monthly to observe any side effect of medication. Clinical manifestation, painless walking distance, Rutherford type and ankle brachial Index (ABI) were studied.

Results: In comparison with control group, the severity of pain, Rutherford type 0 and 1 were improved with statistic significance. Incidence of patients with intermittent claudication decreased from $\mathbf{5 6 . 6 \%}$ before treatment to $28.3 \%$ after treatment; the painless walking distance was prolonged $(116.3 \pm 72.3 \mathrm{~m}$ vs. $243.5 \pm 175.3 \mathrm{~m}, \mathrm{P}<0.001)$; ABI values were increased $(0.74 \pm 0.17$ vs. $0.86 \pm 0.18 ; p<0.001)$. No side effect of medication was observed. Conclusion: Sarpogrelate has a therapeutic effect on patients with atherosclerotic obliterans.
\end{abstract}

Keywords: sarpogrelate hydrochloride, arteriosclerosis obliterans, intermittent claudication

\section{Introduction}

Arteriosclerosis obliterans (ASO) is caused by the arterial atherosclerotic stenosis and obliteration in lower limbs, patients with ASO have discomfort, numbness, intermittent claudication, or even gangrene and ulceration in lower limbs. In recent years, the incidence of

${ }^{1}$ Cardiovascular Surgery, China-Japan Friendship Hospital, the Chinese Ministry of Health, Beijing, China

${ }^{2}$ Department of Anesthesiology, China-Japan Friendship Hospital, the Chinese Ministry of Health, Beijing, China

Received: June 6, 2012; Accepted: October 3, 2012

Corresponding authors: Peng Liu, MD, PhD, Songyi Qian, MD. Cardiovascular surgery, China-Japan Friendship Hospital, No 2, Yinghua yuan East Road, Chaoyang District, Beijing, China Email: ren66sy@gmail.com; liupeng61@yahoo.com.cn; theheartcn@yahoo.com.cn

(C)2013 The Editorial Committee of Annals of Thoracic and Cardiovascular Surgery. All rights reserved.
ASO has a tendency to increase, ${ }^{1,2}$ annual amputation due to ASO is approximately $2 / 10000$ in USA. ${ }^{3)}$ The risk factors of ASO include arterial atherosclerosis, smoking, diabetes mellitus (DM), hyperlipidemia and hypertension. There are no definitive treatments for ASO. The efficacy of surgical treatment, particularly the long-term patency rate of bypass grafts, is not satisfactory, medication is required to maintain the postoperative vascular patency ${ }^{2,4,5)}$ The primary approaches of therapy for ASO are control of risk factors of ASO, such as prohibition of smoking, keeping fit through physical exercise, aggressive management of hyperlipidemia, diabetes and hypertension, etc. ${ }^{1,6)}$

A variety of medications have been used for the treatment of ASO. In recent years, a selective 5-hydoxytryptamine (5-HT)-2A receptor antagonist, sarpogrelate hydrochloride (anplag) has been used for ASO in Asia such as Japan, China. ${ }^{1,37)}$ The physiological role of 5-HT 
through its receptor includes platelet aggregation and vasoconstriction. Sarpogrelate can selectively react with 5-HT receptor, suppress reaction to 5-HT mediated by 5-HT-2A.7) It is reported that sarpogrelate could prevent thrombus formation in experimental animals and inhibit platelet aggregation in patients with ischemic stroke, and has less bleeding events than aspirin. ${ }^{7)}$

As there have been few prospective studies regarding the therapeutic effect of sarpogrelate for Chinese patients with ASO, and the data of clinical efficacy and safety of sarpogrelate in Chinese patients are limited, therefore, we prospectively evaluated the efficacy of sarpogrelate for the treatment of Chinese patients with ASO.

\section{Patients and methods}

The study was approved by our hospital ethical board committee. The informed written consent was received from all patients participating in this study. From January 2010 to April 2012, 176 patients diagnosed with ASO were randomly divided into sarpogrelate group $(n=92)$ and control group $(n=84)$, the randomization was conducted by opening up of a sealed envelope. Patients in sarpogrelate group were administered orally with sarpogrelate $(100 \mathrm{mg}$, tid), whereas patients in control group taken aspirin (100 mg, qd) orally. The assigned medications were blinded to both the investigators and patients.

The main parameters in this study included ankle brachial pressure index (ABPI or ABI) and Rutherford type. The ABI is the ratio of the blood pressure in the lower legs to the blood pressure in the arms, and is calculated by dividing the systolic blood pressure at the ankle by the systolic blood pressures in the arm. ${ }^{8)}$

$$
A B P I_{\text {Leg }}=\frac{P_{\text {Leg }}}{P_{\text {Arm }}}
$$

Where $\mathrm{P}_{\text {leg }}$ is the systolic blood pressure of dorsalis pedis or posterior tibial arteries and $\mathrm{P}_{\text {arm }}$ is the highest of the left and right arm brachial systolic blood pressure.

Whereas the Rutherford classification consists of six categories:9)

1. Mild claudication;

2. Moderate claudication;

3. Severe claudication;

4. Ischemic pain at rest;

5. Minor tissue loss;

6. Major tissue loss.
On measurement of $\mathrm{ABI}$ and walking distance, the related medical staff was blinded to both groups. The treatment course was 12 weeks initiated on the day of the medication. Patients were followed up at 4-week intervals by the vascular specialist. All the relevant clinical manifestation including degree of pain, painless walking distance before claudication, ABI, arterial stenosis and Rutherford type, ${ }^{6)}$ the side effects of medication, especially the hemorrhage after medication were evaluated and documented.

Inclusion conditions included ASO patients, age over 40 years old with risk factor of ASO including long time of smoking, diabetes mellitus (DM) and dyslipidemia, hypertension. Patients had rest pain or intermittent claudication, disappearance or weakness of pulsation of dorsal artery or posterior tibia artery, $\mathrm{ABI}<0.9$. Imaging study including ultrasound, computed tomographic angiography, magnetic resonance angiography or digital subtraction angiography revealed the severe stenosis or occlusion of arteries in the legs, patients had no history of previous surgery on ASO including open surgery or endovascular stenting. Patients were excluded from the study if they have any of the following conditions: previous surgery for ASO either open surgery or endovascular stenting for ASO, history of sensitivity to sarpogrelate or aspirin, active peptic ulcer, history of intracranial hemorrhage, or gastrointestinal hemorrhage, coagulopathy, severe organic diseases such as cardiac, renal, hepatic disorders or malignancy in recent 5 years, patients on wheelchair or being unable to walk, failure to compliance of the assigned medication during the treatment course, refusal to provide written consent to enroll the conservative treatment. To ensure the compliance of patients in both groups with medication, we specifically instructed patients the importance of compliance with medication, if we found or were reported that the medication was changed in schedule or dosage, the patients would be excluded from the study.

Walking distance is defined as painless walking length and was assessed by treadmill test. ABI was measured by the trained certified nurse.

\section{Statistical analysis}

SPSS 13.0 software was used for statistical analysis. Mean \pm SD was used to express the numerical data; student's test was used to compare the ABI before and after treatment. Categorical variables were studied with $\mathrm{X}^{2}$ test. Statistical significance was accepted when $\mathrm{P}$ value was less than 0.05 . 
Table 1 Baseline of patients with ASO

\begin{tabular}{lccc}
\hline & $\begin{array}{c}\text { Sarpogrelate group } \\
\mathrm{n}=92\end{array}$ & $\begin{array}{c}\text { Control group } \\
\mathrm{n}=84\end{array}$ & $P$ value \\
\hline Male & $78(84.78 \%)$ & $74(88.09 \%)$ & 0.522 \\
Age & $64 \pm 11$ & $67 \pm 9$ & 0.051 \\
Smoking & $82(89.13 \%)$ & $78(92.86 \%)$ & 0.390 \\
Years of smoking & $38 \pm 7$ & $35 \pm 8$ & 0.078 \\
Cigarette per day & $42 \pm 31.5$ & $41.5 \pm 21$ & 0.901 \\
Hyperlipidemia & $76(82.61 \%)$ & $62(73.81 \%)$ & 0.156 \\
Hypertension & $72(78.26 \%)$ & $60(71.43 \%)$ & 0.29 \\
DM & $76(82.61 \%)$ & $59(70.24 \%)$ & 0.053 \\
\hline
\end{tabular}

Data are presented as mean \pm SD. DM: diabetes mellitus

Table 2 Change of Rutherford type and ABI after treatment

\begin{tabular}{ccccccccc}
\hline \multirow{2}{*}{$\begin{array}{l}\text { Rutherford } \\
\text { category }\end{array}$} & \multicolumn{3}{c}{ Sarpogrelate group $(\mathrm{n}=92)$} & \multicolumn{2}{c}{ Control group $(\mathrm{n}=84)$} \\
\cline { 2 - 9 } & \multicolumn{2}{c}{ before } & \multicolumn{2}{c}{ after } & \multicolumn{2}{c}{ before } & \multicolumn{2}{c}{ after } \\
\cline { 2 - 9 } & \multicolumn{1}{c}{ No. } & $\%$ & No. & $\%$ & No. & $\%$ & No. & $\%$ \\
\hline 0 & 2 & 2.17 & $38^{* *}$ & 45.23 & 2 & 2.381 & 6 & 7.143 \\
I & 50 & 54.3 & $26^{* *}$ & 30.95 & 43 & 51.19 & 41 & 48.81 \\
II & 22 & 23.9 & 12 & 14.28 & 25 & 29.76 & 22 & 26.19 \\
III & 10 & 10.9 & 8 & 9.52 & 8 & 9.524 & 8 & 9.524 \\
IV & 8 & 8.7 & 8 & 9.52 & 6 & 7.143 & 7 & 8.333 \\
ABI & $0.74 \pm 0.17$ & $0.86 \pm 0.18^{* *}$ & $0.75 \pm 0.14$ & $0.79 \pm 0.17$ \\
\hline
\end{tabular}

$\mathrm{X}^{2}$ test, $* * \mathrm{P}<0.001 ; \mathrm{ABI}$ : ankle brachial index

\section{Results}

The patients with ASO had numbness, pain or cold in the lower limbs, or even intermittent claudication, rest pain, gangrene or ulcer.

The basic conditions of patients with ASO did not differ significantly in both groups in terms of age, gender, risk factors of ASO $(\mathrm{P}<0.05$, Table 1$)$.

In sarpogrelate group, patients reported that the leg pains and painless walking distance were improved after administration of sarpogrelate for 12 weeks. Prior to medication, the number of patients in Rutherford type 0 , I, II, III and IV was 2, 50, 22, 10 and 8, respectively; After medication treatment for 12 weeks, the Rutherford type was improved, the number of patients was 38 in Rutherford type 0, 26 in type I,12 in type II, 8 in type III, and type IV each,of which, there was a significant improvement in patients with type 0 and type $\mathrm{I}(\mathrm{p}<0.001$, Table 2). The number of patients with rest pain was reduced from $22(23.9 \%)$ before therapy to $12(14.28 \%)$ after therapy, even though the difference did not reach to significant ( $p>0.05$, Table 2). The proportion of patients with intermittent claudication was decreased from 54.3\% before treatment to $30.95 \%$ after 12 -week treatment, the walking distance was improved $(116.3 \pm 72.3 \mathrm{~m}$ vs. $243.5 \pm 175.3 \mathrm{~m} ; \mathrm{P}<0.001)$; in contrast, in control group no significant difference was existed before and after treatment ( $p>0.05)$ (Table 2).

The baseline of ABI prior to treatment in both sarpogrelate group and control group was similar with no significant difference $(p>0.05)$. ABI was improved significantly after treatment in sarpogrelate group $(0.74 \pm 0.17$ vs. $0.86 \pm 0.18 ; \mathrm{p}<0.001)$; Whereas no significant change was observed in control group ( $p>0.05$, Table 2).

\section{Discussion}

There is a trend of increasing of incidence of patients with ASO as the economic improvement in China and alteration of Chinese lifestyle, especially well-timed application of investigative facility. Most patients with ASO require medical treatment, and only less than one third of patients are indicated for surgery, those patients included approximately $10 \%$ patients with painless walking distance $<100$ meter or rest pain with ulceration or gangrene, the surgical outcomes have been dismay, because postoperative restenosis of atherosclerotic artery was approximately $30 \% .{ }^{4,5,10)}$ Therefore, the cardinal 
management is medical therapy using anticoagulants, thrombolysis, antiplatelet agents, consecutive alternative biological pressure, etc. ${ }^{11,12)}$ The mechanism of those medications is dilation of blood vessels, anticoagulation, alteration of blood viscosity, improving the collateral circulation. In addition, it is reported that the clinical symptoms could be improved after injecting of automarrow mononuclear cells, however, the survival rate could not be changed. ${ }^{13,14)}$ Naftidrofuryl, buflomedil and pentoxifylline are reported to play the role of dilation of blood vessels, yet they are not available in China.

Sarpogrelate has been widely used in management of ASO for several years in China, yet the prospective observational study is lacking. Our prospective observational study revealed that Rutherford type and ABI in patients with ASO on sarpogrelate have improved significantly in comparison with those taking aspirin only.

Serotonin (5-Hydroxytryptamine, 5-HT) is one of the important neurotransmitters in the central nervous system. A great amount of 5-HT2 are expressed in peripheral tissues of ASO patients, 5-HT participates in vasoconstriction and in aggregation of platelets through 5-HT (2A) receptor (5-HT2AR). ${ }^{1,7,15)}$ Sarpogrelate, a specific antagonist of 5-HT2, can interact with receptor of 5-HT2 on platelets and arterial smooth muscle cells, inhibit the contraction of vessels and adhesion of platelets, and improve the erythrocyte deformability and thereby facilitating the collateral circulation. ${ }^{16,17)}$ The clinical manifestation in our patients treated with sarpogrelate improved, the proportion of patients with intermittent claudication had reduced from $56.6 \%$ to $28.3 \%$, and the walking distance was prolonged significantly.

The principal pathology of ischemic limbs is the local hypoxia caused by poor blood supply to limbs. Sarpogrelate has been reported to increase erythrocyte deformability and inhibit shear stress-induced hemolysis, and has a protective effect on erythrocytes under mechanical shear stress thereby facilitating erythrocytes entering peripheral tissue. ${ }^{18)}$ An oral single-dose administration of sarpogrelate might improve peripheral circulation independent of any changes in blood pressure and heart rate. In our study, the intermittent claudication improved starting 2-5 days after oral administration of sarpogrelate, the rest pains were relieved. Furthermore, we have not observed any side effects of sarpogrelate hydrochloride, which demonstrated the safety of sarpogrelate hydrochloride.

We have to acknowledge that our study is limited by number of participants; painless walking distance before intermittent claudication was reported by patients and these could cause some bias.

In conclusion, our findings indicate that clinical manifestation in patients with ASO receiving sarpogrelate and aspirin improved in terms of painless walking distance prior to intermittent claudication, rest pain and $\mathrm{ABI}$ in comparison with those taking aspirin only, revealing the effect of sarpogrelate on patients with ASO. The randomized large scale trials in multicenter are warranted to confirm its clinical effects.

\section{Disclosure Statement}

This paper is supported in part by National Sciences Foundation of China (Grant No. 81070230). The authors have declared that no competing interests exist. Dr. Ren is the guarantor for this article.

\section{References}

1) Cheng SW, Ting AC, Lau H, et al. Epidemiology of atherosclerotic peripheral arterial occlusive disease in Hong Kong. World J Surg 1999; 23: 202-6.

2) Gao W, Wang F, Liu G, et al. Effectiveness and Safety of Sarpogrelate Hydrochloride for Peripheral Arterial Disease: A Systematic Review. J of Chinese Evidence Medicine 2012; 3: 341-6. (In Chinese)

3) Peacock JM, Keo HH, Duval S, et al. The incidence and health economic burden of ischemic amputation in Minnesota, 2005-2008. Prev Chronic Dis 2011; 8: A141.

4) Komai H, Obitsu Y, Shigematsu H. Diabetes and old age could affect long-term patency of paramalleolar distal bypass for peripheral arterial disease in Japanese patients. Circ J 2011; 75: 2460-4.

5) Saxon RR, Coffman JM, Gooding JM, et al. Longterm patency and clinical outcome of the Viabahn stent-graft for femoropopliteal artery obstructions. J Vasc Interv Radiol 2007; 18: 1341-9; quiz 1350.

6) Lauret GJ, van Dalen HC, Hendriks HJ, et al. When is supervised exercise therapy considered useful in peripheral arterial occlusive disease? A nationwide survey among vascular surgeons. Eur J Vasc Endovasc Surg 2012; 43: 308-12.

7) Miyazaki M, Higashi Y, Goto C, et al. Sarpogrelate hydrochloride, a selective 5-HT2A antagonist, improves vascular function in patients with peripheral arterial disease. J Cardiovasc Pharmacol 2007; 49: 221-7.

8) Al-Qaisi M, Nott DM, King DH, et al. Ankle brachial pressure index (ABPI): An update for practitioners. Vasc Health Risk Manag 2009; 5: 833-41.

9) Norgren L, Hiatt WR, TASC II Working Group, et al. Inter-Society Consensus for the Management of Peripheral Arterial Disease (TASC II). Euro J Vasc Endocasc Surg 2007; 45: S1-75. 
10) De Felice M, Gallo P, Masotti G. Current therapy of peripheral obstructive arterial disease. The non-surgical approach. Angiology 1990; 41: 1-11.

11) Rutherford RB. Clinical staging of acute limb ischemia as the basis for choice of revascularization method: when and how to intervene. Semin Vasc Surg 2009; 22: 5-9.

12) Sultan S, Esan O, Fahy A. Nonoperative active management of critical limb ischemia: initial experience using a sequential compression biomechanical device for limb salvage. Vascular 2008; 16: 130-9.

13) Ruiz-Salmeron R, de la Cuesta-Diaz A, ConstantinoBermejo M, et al. Angiographic demonstration of neoangiogenesis after intra-arterial infusion of autologous bone marrow mononuclear cells in diabetic patients with critical limb ischemia. Cell Transplant 2011; 20: 1629-39.

14) Iafrati MD, Hallett JW, Geils G, et al. Early results and lessons learned from a multicenter, randomized, double-blind trial of bone marrow aspirate concentrate in critical limb ischemia. J Vasc Surg 2011; 54: 1650-8.

15) Yoshimasu T, Ikeda T, Uede K, et al. Effects of sarpogrelate hydrochloride on skin ulcers and quality of life in patients with systemic sclerosis. J Dermatol 2012; 39: 536-40.

16) Kokubu N, Tsuchihashi K, Yuda S, et al. Persistent insulin-sensitizing effects of sarpogrelate hydrochloride, a serotonin $2 \mathrm{~A}$ receptor antagonist, in patients with peripheral arterial disease. Circ J 2006; 70: 1451-6.

17) Satoh K, Yatomi Y, Ozaki Y. A new method for assessment of an anti-5HT(2A) agent, sarpogrelate hydrochloride, on platelet aggregation. J Thromb Haemost 2006; 4: 479-81.

18) Nakamura K, Kawahito K. Erythrocyte-protective effect of sarpogrelate hydrochloride (Anplag (), a selective 5-HT2 receptor antagonist: an in vitro study. J Artif Organs 2010; 13: 178-81. 